\title{
Long-term oxygen therapy: Are we prescribing appropriately?
}

\author{
$M^{a}$ Rosa Güell Rous \\ Departament de Pneumologia, \\ Hospital de la Santa Creu I de Sant \\ Pau, Barcelona, Spain
}

Correspondence: Ma Rosa Güell Rous Departament de Pneumologia, Hospital de la Santa Creu I de Sant Pau, Sant Antoni $\mathrm{M}^{\mathrm{a}}$ Claret, 167, 08025 Barcelona, Spain

Tel +34935565972

Fax+3493556560I

Email mguellr@santpau.es

\begin{abstract}
Long-term oxygen therapy (LTOT) is the treatment proven to improve survival in chronic obstructive pulmonary disease (COPD) patients with chronic respiratory failure. It also appears to reduce the number of hospitalizations, increase effort capacity, and improve health-related quality of life. Standard LTOT criteria are related to COPD patients who have $\mathrm{PaO}_{2}<60 \mathrm{mmHg}$, are in a clinical stable situation, and are receiving optimal pharmacological treatment. According to LTOT guidelines, oxygen should be prescribed for at least 18 hours per day although some authors consider 24 hours would be more beneficial. The benefits of LTOT depend on correction of hypoxemia. Arterial blood gases should be measured at rest. During exercise, an effort test should be done to assure adequate $\mathrm{SaO}_{2}$. During sleep, continuous monitoring of $\mathrm{SaO}_{2}$ and $\mathrm{PaCO}_{2}$ should be performed to confirm correction of $\mathrm{SaO}_{2}$ overnight. An arterial blood gas sample should be taken at awakening to assess $\mathrm{PaCO}_{2}$ in order to prevent hypoventilation from the oxygen therapy. Several issues that need to be addressed are the use of LTOT in COPD patients with moderate hypoxemia, the efficacy of LTOT in patients who desaturate during exercise or during sleep, the optimal dosage of oxygen supplementation, LTOT compliance, and the LTOT prescription in diseases other than COPD.
\end{abstract}

Keywords: long-term oxygen therapy, COPD, oxygen supplementation, chronic respiratory failure, hypoxemia

\section{Benefits of long-term oxygen therapy}

Early reports on respiratory failure in patients with chronic obstructive pulmonary disease (COPD) suggested that oxygen therapy decreases pulmonary hypertension and red cell mass while simultaneously increasing exercise capacity (O’Donohue 1995) In the 1980s, two landmark multicentre trials, the Nocturnal Oxygen Therapy Trial (NOTT) (NOTT 1980) and the Medical Research Council (MRC) (MRC 1981) study, showed that long-term oxygen therapy (LTOT) was the sole treatment that improved survival in patients with COPD and chronic respiratory failure (CRF), and that these patients received oxygen for at least 18 hours/day (Figure 1). These findings were reaffirmed in later trials (Dubois et al 1994; Zielinski et al 1998). NOTT (1980) and MRC (1981) trials led to the establishment of criteria for oxygen therapy in almost every country in the developed world (Table 1) (Sanchez-Agudo et al 1998; ATS 1995; Siafakas et al 1995; Celli et al 2004; Rabe et al 2007). There is also evidence that LTOT has benefits other than survival. These include stabilization of pulmonary arterial hypertension, fewer cardiac arrhythmias and electrocardiographic findings suggestive of myocardial ischemia, increased exercise capacity, an improvement in neuropsychiatric function and in healthrelated quality of life, and a reduction of exacerbations or hospitalizations (Tirlapur and Mir 1982; Davidson et al 1988; Morrison and Stovall 1992; Dean et al 1992; Clini et al 1996; Wedzicha 2000; Ringbaek et al 2002; Eaton et al 2004). However, these clinical benefits depend on treatment compliance, the duration of the treatment, and adequate 


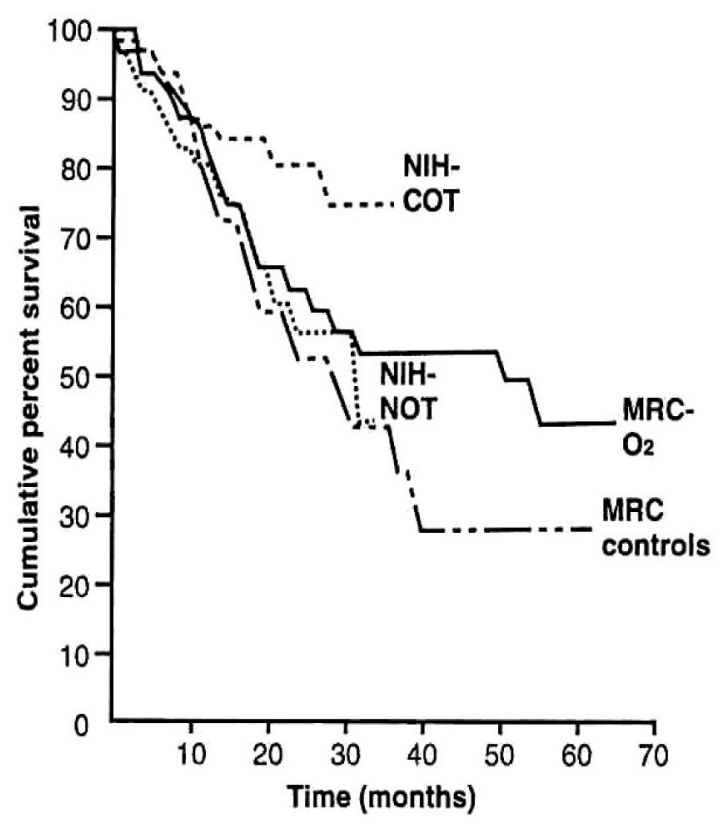

Figure I Comparison of survival curves of NOTT and MRC studies. The poorest prognosis was in the MRC controls who received no oxygen. The best survival was in the NOTT patients who received oxygen for >19 hours/day. Copyright (c) 1980, 198I. Modified with permission from [NOTT] Nocturnal Oxygen Therapy Trial Group. 1980. Continuous or nocturnal oxygen therapy in hypoxemic chronic obstructive lung disease: a clinical trial. Nocturnal Oxygen Therapy Trial Group. Ann Inter Med, 93:39I-8; [MRC] Medical Research CouncilWorking Party. 198I. Long term domiciliary oxygen therapy in chronic hypoxic cor pulmonale complicating chronic bronchitis and emphysema. Report of the Medical Research Council Working Party. Lancet, I:68I-5.

Abbreviations: MRC, Medical Research Council; NIH, National Institutes of Health; NOTT, Nocturnal Oxygen Therapy Trial.

correction of hypoxemia (Gorecka et al 1992; Sliwinski et al 1994; Pepin et al 1996; Plywaczewski et al 2000; Criner 2000; Tárrega et al 2002).

\section{Which patients can improve with LTOT?}

The NOTT and MRC studies demonstrated that LTOT improved survival in COPD with resting partial pressure of oxygen in arterial blood $\left(\mathrm{PaO}_{2}\right)<60 \mathrm{mmHg}$ either with or without hypercapnia. The trial reported by Gorecka and colleagues (1997), however, found LTOT had no effect on survival in patients with COPD and moderate hypoxemia (56-65 mmHg). Does the lack of benefits on survival in some patients mean that not all these patients should be offered LTOT? Or should we analyze other benefits of LTOT rather than survival? From the results of many trials it is reasonable to hypothesize that LTOT may benefit COPD patients in different ways, such as in exercise capacity (Davidson et al 1988; Morrison and Stovall 1992; Dean et al 1992), health-related quality of life (Eaton et al 2004), exacerbations or hospitalizations (Clini et al 1996; Ringbaek et al 2002). Therefore, as the recent National Heart, Lung, and Blood Institute (NHLBI) workshop proposed, we need to consider these other benefits in COPD patients independently from the increase in survival (Croxton and Bailey 2006).

\section{How to prescribe LTOT?}

Supplemental oxygen should be included in the continuum of care along with cessation of smoking, pharmacological therapy, vaccination, attention to infectious exacerbations, and respiratory rehabilitation. Good coping skills, motivation, and a supportive family influence compliance with supplemental oxygen. Patients need to be able to meet the criteria for LTOT (Table 1); they must be clinically stable, and should be receiving optimal pharmacological treatment prior to commencement. The oxygen flow should be titrated during the daytime with the patient awake and at rest. It is recommended to set the oxygen flow to achieve transcutaneus oxygen saturation $\left(\mathrm{SaO}_{2}\right) \geq 90 \%$.

\section{Are we assessing eligibility appropriately for LTOT?}

Before prescribing LTOT, arterial blood gas measurements should be performed at least twice to confirm the patient meets the criteria for this treatment. However, in clinical practice, LTOT is frequently prescribed in an unstable clinical situation or after a single assessment, as reported by several authors. Levi-Valensi and colleagues (1986) found that 23 of 77 patients under LTOT since hospital discharge were no longer eligible at 3 months' follow up. More recently, in a randomized trial, Guyatt and colleagues (2005) affirmed that reassessment of applicants for LTOT identified an appreciable portion of initially eligible patients who were no longer eligible.

\section{Should all COPD patients with criteria for LTOT be treated 24 hours/day?}

Although different guidelines recommend LTOT for at least 18 hours/day, the combined data from the NOTT (1980) and MRC (1981) studies indicate that the longer the oxygen-use, the greater the benefit. Careful observation of data in Figure 1 reveals that patients who used oxygen 24 hours/day have higher survival than the other groups. Some studies have shown that COPD patients have severe desaturation during daily activities such as walking, eating, or washing (Soguel Schenkel et al 1996). The NOTT and MRC studies were developed before portable oxygen devices were in common use; liquid oxygen devices are now readily available in most developed countries. 
Table I Indications for LTOT in COPD (Cooper 1993)

\section{UNITED STATES}

$\mathrm{PaO}_{2}<55 \mathrm{mmHg}$ or $\mathrm{SaO}_{2}<88 \%$ (room air)

$\mathrm{PaO}_{2}$ 56-59 mmHg or $\mathrm{SaO}_{2} 89 \%-90 \%$, with (one or more)

Pulmonary hypertension

Evidence of cor pulmonale or edema due to heart failure

Elevated hematocrit $(>56 \%)$

\section{UNITED KINGDOM}

I. COPD (FEV $<1.5 \mathrm{~L}, \mathrm{FVC}<2.0 \mathrm{~L})$, hypoxemia $\left(\mathrm{PaO}_{2}<55 \mathrm{mmHg}\right)$, hypercapnia $\left(\mathrm{PaCO}_{2}>45 \mathrm{mmHg}\right)$, and edema. Stability demonstrated over 3 weeks

2. As in I but without edema or $\mathrm{PaCO}_{2}>45 \mathrm{mmHg}$ Palliative therapy may be prescribed

\section{EUROPE}

I. $\mathrm{PaO}_{2}<55 \mathrm{mmHg}$, steady-state COPD

2. $\mathrm{PaO}_{2} 55-65 \mathrm{mmHg}$ with (one or more)

Pulmonary hypertension

Evidence of cor pulmonale or edema due to heart failure

Elevated hematocrit $(>56 \%)$

Restrictive disease with $\mathrm{PaO}_{2}<55 \mathrm{mmHg}$

\section{AUSTRALIA}

I. $\mathrm{PaO}_{2}<56 \mathrm{mmHg}$, COPD, Right ventricular hypertrophy, polycythemia, and edema

2. Desaturation $<90 \%$ on exercise

3. Refractory dyspnea associated with cardiac failure

Abbreviations: COPD, chronic obstructive pulmonary disease; FEV , forced expiratory volume in one second; $\mathrm{FVC}$, forced vital capacity; $\mathrm{PaO}_{2}$, partial pressure of oxygen in arterial blood; $\mathrm{SaO}_{2}$, oxygen saturation.

It may therefore be advisable to prescribe LTOT in the form of liquid oxygen and advice patients to use this therapy all day, especially during exercise. Trials investigating the benefits of 24-hour rather than 18-hour LTOT are needed.

\section{Does the oxygen flow prescribed adequately correct saturacion of oxygen?}

\section{At rest}

According to COPD and LTOT treatment guidelines (SanchezAgudo et al 1998; ATS 1995; Siafakas et al 1995; Celli et al 2004; Rabe et al 2007) the oxygen flow should be titrated during the daytime when the patient is clinically stable and awake, using the same oxygen source as that received at home. It is recommended to set the oxygen flow to achieve $\mathrm{SaO}_{2} \geq 90 \%$ using a pulse oximeter, taking an arterial blood gas sample to assure correct titration. An overnight pulse oximetry should be recorded during sleep to evaluate the correction in nocturnal $\mathrm{SaO}_{2}$.

From an international survey about LTOT prescription, Wijkstra and colleagues (2001) showed that there were marked differences among countries as to how LTOT was prescribed. In many European countries patients are tested in supine rather than sitting position, the target $\mathrm{SaO}_{2}$ varies from $90 \%$ to $92 \%$, and not all countries measure arterial blood gases or test $\mathrm{SaO}_{2}$ during sleep.

\section{During exercise}

There are no specific recommendations about oxygen prescription during exercise. Some guidelines advise using an effort test to assess the adequate oxygen flow to correct oxygen desaturation. To establish the appropriate oxygen flow it is recommended to test patients under increasing oxygen flow until $\mathrm{SaO}_{2} \geq 90 \%$. The most frequently used effort test is the 6-minute walking test $(6 \mathrm{Wt})$, which some authors have shown to be a reliable measurement. Comparing $\mathrm{SaO}_{2}$ during the $6 \mathrm{WT}$ with that of ambulatory pulsioximetry, Morante and colleagues (2005) showed that the 6WT is a good reflection of desaturation during daily activities measured by ambulatory pulse oximetry. These authors also demonstrated a good correlation between the two measurements when patients are breathing oxygen $(\mathrm{p}<0.01)$ (Tables 2 and 3$)$.

Wijkstra and colleagues' (2001) international survey showed that most countries assess patients during exercise to achieve $\mathrm{SaO}_{2} \geq 90 \%$. However, ATS guidelines and some European countries recommend the resting flow rate should be increased by $11 . \mathrm{min}^{-1}$ or that the same flow rate as at rest should be used without testing the oxygen flow rate needed during exercise.

\section{During sleep}

There are three ways to adjust the oxygen flow during sleep: maintaining the resting flow rate, increasing the resting flow rate by 1 or $2.1 . \mathrm{min}^{-1}$, or setting the flow rate according to continuous monitoring of $\mathrm{SaO}_{2}$ to maintain $\mathrm{SaO}_{2} \geq 90 \%$.

Using the resting flow rate, several studies (Plywaczewski et al 2000; Tárrega et al 2002, Nisbet et al 2006) have found an inadequate correction in nocturnal $\mathrm{SaO}_{2}$ in a high, and variable percentage of patients $(13 \%-48 \%)$. Various factors

Table 2 comparison of $\mathrm{SaO}_{2}$ under liquid oxygen between the 6 minute walking test (6WT) and the ambulatory pulse oximetry (AP)

\begin{tabular}{llll}
\hline & 6WT & AP & (p) \\
\hline $\mathrm{SaO}_{2}$ mean (\%) & $90(3)$ & $92(2)$ & 0.28 \\
$\mathrm{CT90}(\%)$ & $53(35)$ & $22(15)$ & 0.47 \\
$\mathrm{CT} 88(\%)$ & $29(33)$ & $11(11)$ & 0.1 \\
$\mathrm{CT} 85(\%)$ & $8(11)$ & $6(6)$ & 0.6 \\
\hline
\end{tabular}

Abbreviations: Mean $\mathrm{SaO}_{2}$, mean value of saturation of oxihemoglobin; CT90, percentage of time with $\mathrm{SaO}_{2}<90 \%$; CT88, percentage of time with $\mathrm{SaO}_{2}<88 \%$; CT85, percentage of time with $\mathrm{SaO}_{2}<85 \%$ (Morante et al 2005). 
Table 3 Correlation of the $\mathrm{SaO}_{2}$ values between the 6-minute walking test (6WT) and the ambulatory pulse oximetry (AP)

\begin{tabular}{llll}
\hline & 6WT & AP & $\boldsymbol{r}(\mathbf{p})$ \\
\hline $\mathrm{SaO}_{2}$ mean (\%) & $84(7)$ & $89(4)$ & $0.7(<0.0 \mathrm{I})$ \\
$\mathrm{CT} 9$ (\%) & $77(34)$ & $53(32)$ & $0.6(<0.0 \mathrm{I})$ \\
$\mathrm{CT} 88(\%)$ & $56(42)$ & $35(30)$ & $0.5 \mathrm{I}(<0.0 \mathrm{I})$ \\
$\mathrm{CT} 85(\%)$ & $42(44)$ & $19(23)$ & $0.65(<0.0 \mathrm{I})$ \\
\hline
\end{tabular}

Abbreviations: Mean $\mathrm{SaO}_{2}$, mean value of saturation of oxihemoglobin; $\mathrm{CT} 90$, percentage of time with $\mathrm{SaO}_{2}<90 \%$; $\mathrm{CT} 88$, percentage of time with $\mathrm{SaO}_{2}<88 \%$; CT85, percentage of time with $\mathrm{SaO}_{2}<85 \%$ (Morante et al 2005).

could explain these differences. Firstly, neither of the two first studies (Plywaczewski et al 2000; Tárrega etal 2002) excluded diseases associated to COPD, such as pulmonary restrictive disorders, that could produce nocturnal desaturation. Secondly, the presence of obstructive sleep apnea syndrome (OSAS) was not ruled out.

To prevent sleep desaturation, some guidelines recommend increasing the resting flow rate oxygen 1 or 2.1. $\mathrm{min}^{-1}$. In their international study, Wijkstra and colleagues (2001) observed that in Canada and US there was a tendency to increase the oxygen flow rate during sleep, whereas in most European countries the tendency was either to maintain the same flow rate as that used during the day or to test patients individually.

Oxygen therapy can generate several potentially detrimental effects. The most relevant of these is hypercapnia, which is mediated by mechanisms such as hypoventilation, ventilation-perfusion redistribution, or the Haldane effect. Moreover, sleep itself generates ventilatory alterations that include a decrease in baseline metabolism, an increase in airway resistance, hypotonia of the respiratory pump, and decreased sensitivity of respiratory centers. In healthy individuals, these alterations generate only small changes in $\mathrm{PaO}_{2}$ or $\mathrm{PaCO}_{2}$ during the night, but in COPD patients they may cause severe hypoxemia and hypercapnia. In this population, physiopathologic phenomena such as greater hypoventilation due to a reduction in tidal volume, greater alterations in ventilation-perfusion distribution, associated OSAS and reduction of mucociliar clearance may also be key to increased hypercapnia and development of sleep hypoventilation (SH). O'Donoghue and colleagues (2003) and Tárrega and colleagues (2002) observed SH in a high percentage of COPD patients, $43 \%$ and $59 \%$, respectively, and suggested the finding was likely due to the oxygen therapy. Increasing the resting flow rate by 1 or $21 \mathrm{~min}^{-1}$ may therefore exacerbate hypoventilation. Our group recently conducted a study to evaluate changes in gas exchange. We increased the nocturnal flow rate by 1 liter and observed that this induced SH and respiratory acidosis in a significant number of patients who did not have SH when they received the same oxygen titration as during the day (34.2\% vs. $23.7 \%$ of patients) (Figures 2 and 3) (Samolski et al in press). This finding further supports the hypothesis that oxygen can induce hypoventilation. Plant and colleagues (2000) had previously shown that a high oxygen flow rate in acute respiratory failure in COPD patients was the most frequent cause of hypercapnia and led to the need for noninvasive ventilation. Such results reinforce the need to evaluate and titrate the nocturnal oxygen flow individually, at least in hypercapnic COPD patients.

According to some guidelines, continuous monitoring of $\mathrm{SaO}_{2}$ should be performed to set the oxygen flow rate during sleep to maintain $\mathrm{SaO}_{2} \geq 90 \%$ overnight. We consider arterial blood gases should be measured early in the morning to test for hypercapnia and respiratory acidosis. If patients present these findings then our advice is to test for the presence of OSAS. Once OSAS is ruled out, the best way to treat these patients with SH under oxygen therapy may be to use the Venturi mask or noninvasive ventilation. The subgroup of COPD patients who develop SH under oxygen therapy may be the only COPD candidates for whom domiciliary noninvasive ventilation therapy is recommended rather than LTOT.

\section{What is the role of oxygen conservation systems on COPD patients undergoing LTOT?}

Oxygen conservation systems could optimize the benefit of LTOT in some patients. Such devices should only be used in patients who need low oxygen flow rates. Their effectiveness depends on the valve trigger, the patient's breathing rate, and the presence of nasal breathing.

Some studies have shown that conservation systems are effective at rest, during sleep and during exercise (Tiep et al 1987, 2002; Bower et al 1988; Braun et al 1992; Cuvelier et al 1999; Garrod et al 1999; Fuhrman et al 2004). Cuvelier and colleagues (1999) showed that there were no differences in the ventilatory parameters when COPD patients used conventional or conservation systems for LTOT. Other studies, however, have shown that to maintain an adequate $\mathrm{SaO}_{2}$ in some patients it is necessary to increase the oxygen flow rate. Conservative systems have different characteristics. Bliss and colleagues (2004) compared three different models of these systems and found that each model gave a different quantity of 


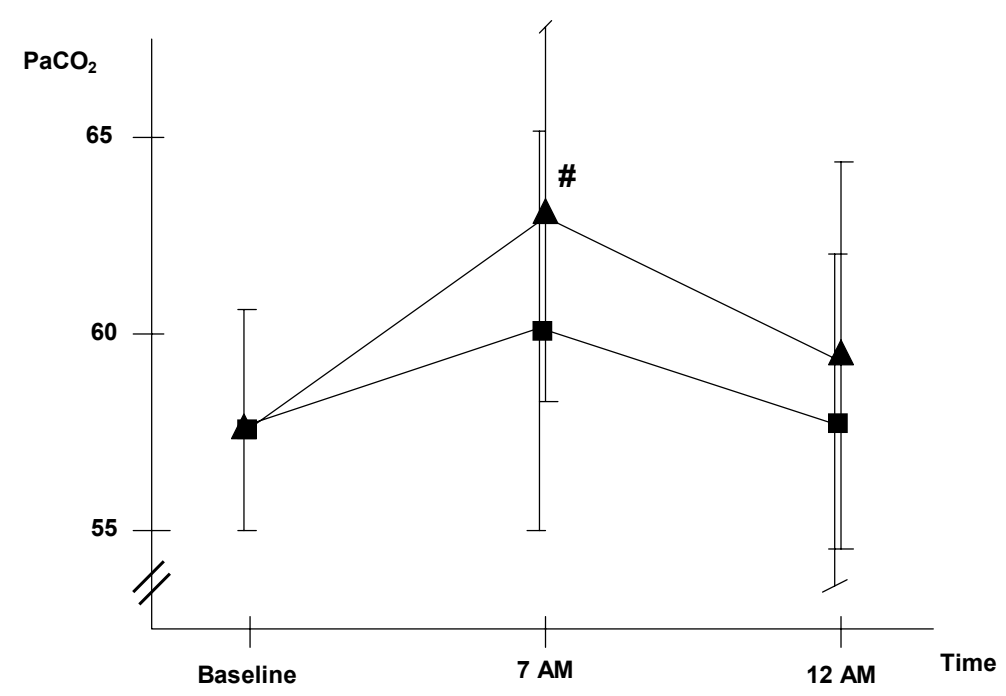

Figure 2 Evolution of $\mathrm{PaCO}_{2}$ : oxygen flow titrated at daytime, $\mathbf{\Lambda}$ : I litre increase in oxygen flow.

Note: $\mathrm{p}<0.05$ Samolski et al in press.

Abbreviation: $\mathrm{PaCO}_{2}$, partial pressure of oxygen in arterial blood.

oxygen. These systems should be tested individually at rest and during exercise.

\section{Recommendations for future research}

The NHLBI workshop report (Croxton and Bailey 2006) recently reported a very interesting review about the current state of knowledge of LTOT and proposed topics for future research. The most relevant issues for study, according to the said paper, are the use of LTOT in COPD patients with moderate hypoxemia, the efficacy of LTOT in patients who desaturate during exercise and sleep, the optimal dosage of oxygen supplementation, and the compliance of LTOT.

Three considerations related to LTOT for COPD patients with moderate hypoxemia are yet to be addressed. The first of these is that the duration of treatment may need to be longer than 18 hours per day. Second, benefits other than survival should be considered, such as health-related quality of life, exercise capacity, or hospitalizations. Third, it is necessary to identify subgroups of COPD patients with moderate hypoxemia who can benefit from LTOT, such as patients with pulmonary hypertension, low body mass index,

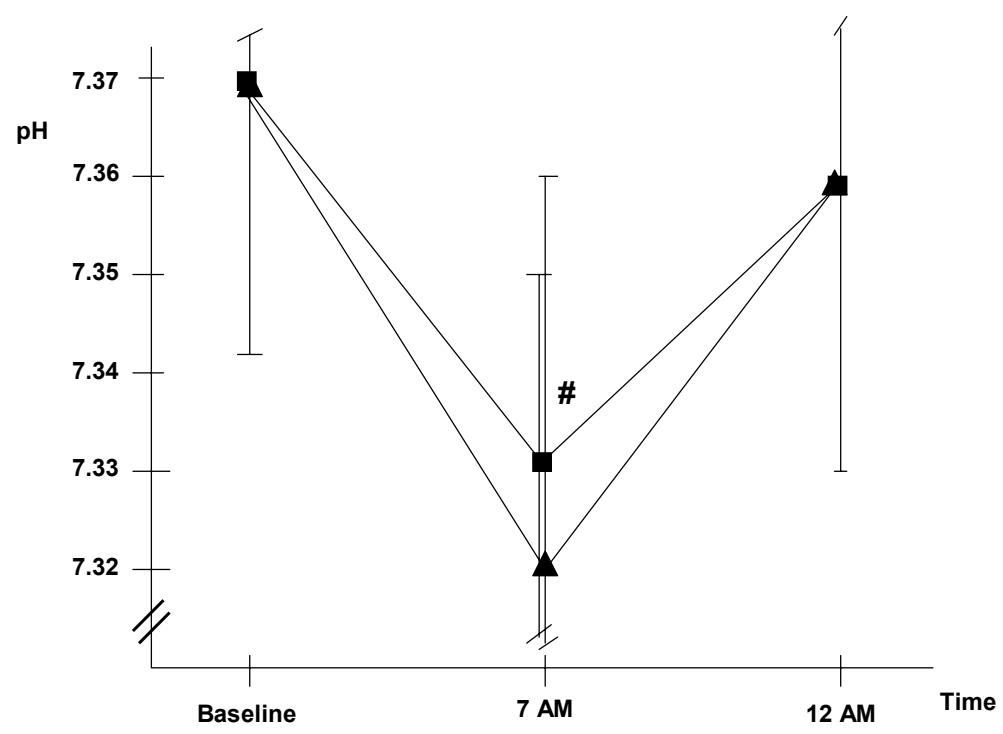

Figure 3 Evolution of $\mathrm{pH}$ : oxygen flow titrated at daytime, $\mathbf{A}$ : I litre increase in oxygen flow.

Note: $p<0.05$ Samolski et al in press. 
poor exercise capacity, frequent exacerbations, or co-morbid cardiac disease.

There is insufficient evidence that LTOT can provide benefits to patients with exercise desaturation. Nevertheless, it seems logical that episodic hypoxemia may itself have adverse consequences and increase effort dyspnoea. Very few studies have been performed to date regarding the role of oxygen therapy for COPD patients with sleep desaturation. We can hypothesize that oxygen therapy could increase survival by reducing the risk factors, especially cardiac events, produced by desaturation during sleep, and also increase the quality of sleep. From the NOTT and MRC studies it seems obvious that 24 hours/day will be much more beneficial for COPD patients than 18 hours/day, and that an adequately adjustment of oxygen flow at rest, during effort, and sleep will provide more benefits.

Finally, it is well known that compliance with LTOT is very poor in COPD patients. An important goal in the future therefore should be to develop patient education strategies regarding the use of LTOT and to improve oxygen therapy devices.

Based on these observations concerning LTOT, the NHLBI workshop (Croxton and Bailey 2006) proposed four trials to investigate the role of: (1) oxygen supplementation during ambulation; (2) continuous oxygen supplementation for moderate hypoxemia; (3) nocturnal oxygen treatment for desaturation during sleep; and (4) detailed, individualized prescriptions for LTOT supplementation. We would like to add a further topic, addressed to the prescription of LTOT in patients with diseases other than COPD, such as restrictive pulmonary diseases or cardiac diseases.

\section{References}

[ATS] American Thoracic Society. 1995. Standards for the diagnosis and care of patients with chronic obstructive pulmonary disease. American Thoracic Society. Am J Resp Crit Care Med, 152(5 Pt 2):S77-121.

Bliss P, McCoy RW, Adams AB. 2004. Characteristics of demand oxygen delivery systems: maximun output and setting recommendations. Respir Care, 49:160-5.

Bower JS, Brook CJ, Zimmer K, et al. 1988. Performance of a demand oxygen saver system during rest, exercise, and sleep in hypoxemic patients. Chest, 94:77-80.

Braun SR, Spratt G, Scott GC, et al. 1992. Comparision of six oxygen delivery systems for COPD patients in rest and exercise. Chest, 102:694-8.

Celli BR, MacNee W; ATS/ERS Task Force. 2004. Standards for the diagnosis and treatment of patients with COPD: a summary of the ATS/ERS position paper. Eur Respir J, 23:932-46.

Clini E, Vitacca M, Foglio K, et al. 1996. Long-term home care programmes may reduce hospital admissions in COPD with chronic hypercapnia. Eur Respir J, 9:1605-10.

Cooper CB. 1993. Long-term oxygen therapy. In: Casaburi R, Petty TL (eds). Principles and Practice of Pulmonary Rehabilitation. Philadelphia: WB Saunders Company, pp. 183-203.

Criner GJ. 2000. Effects of long-term oxygen therapy on mortality and morbidity. Respir Care, 45:105-18.
Croxton TL, Bailey WC; for the NHLBI Working Group on Long-Term Oxygen Therapy in COPD. 2006. Long-term oxygen treatment in chronic obstructive pulmonary disease: Recommendations for future research. Am J Respir Crit Care Med, 174:373-8.

Cuvelier A, Muir JF, Czernichow P, et al. 1999. Nocturnal efficiency and tolerance of a demand oxygen delivery system in COPD patients with nocturnal hypoxemia. Chest, 116:22-9.

Davidson AC, Leach R, George RJD, et al. 1988. Supplemental oxygen and exercise ability in chronic obstructive airways disease. Thorax, 43:965-71.

Dean NC, Brown JK, Himelman RB, et al. 1992. Oxygen may improve dyspnea and endurance in patients with chronic obstructive pulmonary disease and only mild hypoxemia. Am Rev Respir Dis, 146:941-5.

Dubois P, Jamart J, Machiels J, et al. 1994. Prognosis of severely hypoxemic patients receiving long-term oxygen therapy. Chest, 105:469-74.

Eaton T, Lewis C, Young P, et al. 2004. Long-term oxygen therapy improves Health-related quality of life. Respir Med, 98:285-93.

Siafakas NM, Vermeire P, Pride NB, et al. 1995. Optimal assessment and management of chronic obstructive pulmonary disease (COPD). The European Respiratory Society Task Force. Eur Respir J, 8:1398-420.

Fuhrman C, Chouaid C, Herigault R, et al. 2004. Comparison of four demand oxygen delivery systems at rest and during exercise for chronic obstructive pulmonary disease. Respir Med, 98:938.44.

Garrod R, Bestall JC, Paul E, et al. 1999. Evaluation of pulsed dose oxygen delivery during exercise in patients with severe chronic obstructive pulmonary disease. Thorax, 54:242-44.

Gorecka D, Gorzelak K, Sliwinski P, et al. 1997. Effect of long term oxygen therapy on survival in patients with chronic obstructive pulmonary disease with moderate hypoxemia. Thorax, 52:674-9.

Gorecka D, Sliwinski P, Zielinski J. 1992. Adherence to entry criteria and one year experience of long-term oxygen therapy in Poland. Eur Respir J, 5:848-52.

Guyatt GH, Nonoyama M, Lacchetti CH, et al. A randomized trial of strategies for assessing eligibility for long-term domiciliary oxygen therapy. Am J Respir Crit Care Med, 172:573-80.

Levi-Valensi P, Weitzenblum E, Pedinielli JL, et al. 1986. Three month follow up of arterial blood gas determinations in candidates for long term oxygen therapy. Am Rev Respir Dis, 133:547-51.

Morante F, Güell R, Mayos M. 2005. Eficacia de la prueba de 6 minutos de marcha en la valoración de la oxigenoterapia de deambulación. Arch Bronconeumol, 41:596-600.

Morrison DA, Stovall JR. 1992. Increased exercise capacity in hypoxemic patients after long-term oxygen therapy. Chest, 102:542-50.

[MRC] Medical Research Council Working Party. 1981. Long term domiciliary oxygen therapy in chronic hypoxic cor pulmonale complicating chronic bronchitis and emphysema. Report of the Medical Research Council Working Party. Lancet, 1:681-5.

Nisbet M, Eaton T, Lewis C, et al. 2006. Overnight prescription of oxygen in long-term oxygen therapy: time to reconsider the guidelines? Thorax, 61:779-82.

[NOTT] Nocturnal Oxygen Therapy Trial Group. 1980. Continuous or nocturnal oxygen therapy in hypoxemic chronic obstructive lung disease: a clinical trial. Nocturnal Oxygen Therapy Trial Group. Ann Inter Med, 93:391-8.

O'Donoghue F, Catcheside P, Ellis E, et al. 2003. Sleep hypoventilation in hypercapnic chronic obstructive pulmonary disease: prevalence and associated factors. Eur Respir J, 21:977-84.

O'Donohue WJ. 1995. Long-Term Oxygen Therapy. Scientific Basis and Clinical Application (Lung Biology in Health and Disease). Vol. 81. New York: Marcel Dekker Inc.

Pepin JL, Barjhoux E, Deschaux CH, et al. 1996. Long-term oxygen therapy at home. Compliance with medical prescription and effective use of therapy. Chest, 109:1144-50.

Plant PK, Owen JL, Elliott MW. 2000. One-year period prevalence study of respiratory acidosis in acute exacerbations of COPD: implications for the provision of non-invasive ventilation and oxygen administration. Thorax, 55:550-4. 
Plywaczewski R, Sliwinski P, Nowinski A, et al. 2000. Incidence of nocturnal desaturation while breathing oxygen in COPD patients undergoing long-term oxygen therapy. Chest, 117:679-87.

Rabe KF, Hurd S, Anzueto A, et al. Global Initiative for Chronic Obstructive Lung Disease. 2007. Global strategy for the diagnosis, management, and prevention of chronic obstructive pulmonary disease: GOLD executive summary. Am J Respir Crit Care Med, 176:532-55.

Ringbaek TJ, Viskim K, Lange P. 2002. Does long-term oxygen therapy reduce hospitalisations in hypoxaemic chronic obstructive pulmonary disease? Eur Respir J, 20:38-42.

Samolski D, Tárrega J, Antón A, et al. Sleep hypoventilation due to an increase in nocturnal oxygen flow in hypercapnic COPD patients undergoing long-term oxygen therapy. In press.

Sanchez-Agudo L, Cornudella R, Estopá R, et al. 1998. Normativa para la indicación y empleo de la oxigenoterapia continuada nocturna. Arch Bronconeumol, 34:87-94.

Sliwinski P, Lagosz M, Gorecka D, et al. 1994. The adequacy of oxygenation in COPD patients undergoing long-term therapy assessed by pulse oximetry at home. Eur Respir J, 7:274-8.

Soguel Schenkel N, Burdet L, de Muralt D, et al. 1996. Oxygen saturation during daily activities in Chronic obstructive pulmonary disease. Eur Respir J, 9:2584-9.
Tárrega J, Güell R, Antón A, et al. 2002. Are daytime arterial blood gases a good reflection of nighttime gas exchange in patients on long-term oxygen therapy? Respir Care, 47:882-6.

Tiep BL, Barnett J, Schiffman G, et al. 2002. Maintaning oxygenation via demand oxygen delivery during rest and exercise. Respir Care, 47:887-92.

Tiep BL, Carter R, Nicotra B, et al. 1987. Demand oxygen delivery during exercise. Chest, 91:15-20.

Tirlapur VG, Mir MA. 1982. Nocturnal hypoxemia and associated electrocardiographic changes in patients with chronic obstructive airways disease. $N$ Engl J Med, 306:125-30.

Wedzicha J. 2000. Effects of long-term oxygen therapy on neuropsychiatric function and quality of life. Respir Care, 45:119-24.

Wijkstra PJ, Guyatt GH, Ambrosino N, et al. 2001. International approaches to the prescription of long-term oxygen therapy. Eur Respir J, 18:909-13.

Zielinski J, Tobiasz M, Hawrylkiewicz I, et al. 1998. Effects of long-term oxygen therapy on pulmonary hemodynamics in COPD patients. Chest, 113:65-70. 
